\title{
Four-Wave Mixing and Bragg Scattering in Resonant Seed Modulation Instability in Optical Fiber
}

\author{
Duc Minh Nguyen ${ }^{1,2}$, Di Yang ${ }^{3,4}$, Cesare Soci ${ }^{2}$, Xuan Quyen Dinh ${ }^{1,5}$, Ming Tang ${ }^{4}$ and Ping Perry Shum ${ }^{1,3}$ \\ ${ }^{l}$ CINTRA CNRS/NTU/THALES, UMI 3288, Research Techno Plaza, 50 Nanyang Drive, Singapore \\ ${ }^{2}$ Centre for Disruptive Photonic Technologies, Nanyang Technological University, Singapore \\ ${ }^{3}$ OPTIMUS, Photonics Centre of Excellence, Nanyang Technological University, Singapore \\ ${ }^{4}$ NGIA, Huazhong University of Science and Technology, China \\ ${ }^{5}$ Thales Solutions Asia Pte. Ltd., R\&T Centre, 28 Changi North Rise, Singapore 498755 \\ dmnguyen@ntu.edu.sg
}

\begin{abstract}
We give a physical interpretation of the dynamics of resonant seed modulation instability of quasi-continuous picosecond pulses in terms of cascaded four-wave mixing and Bragg scattering in fiber frequency combs. We also report a critical condition to excite the resonant seeding effect.

OCIS codes: (320.6629) Supercontinuum generation; (190.4380) Nonlinear optics, four-wave mixing.
\end{abstract}

\section{Introduction}

Supercontinuum (SC) generation in an optical fiber has recently drawn significant interest due to its applications in low noise broadband sources as well as in the context of its fundamental link with the emergence of giant "rogue wave" instabilities on the ocean [1]. In the anomalous dispersion regime, SC generation is initiated by modulation instability (MI) and active control of the MI process is therefore important.

It has been demonstrated that seeding a pump with an extremely weak co-propagating wave is an efficient way to enhance the spectral bandwidth and noise properties of MI-driven SC generation [2, 3]. This seeding effect has been confirmed numerically and experimentally for both strong coherence [2] and partial coherence between seed and pump sources [3]. However, a clear physical interpretation of the seeding effect on a quasi-continuous picoseconds pulse has not yet been given, except for being known that it results from the initial modulation on the pump caused by the seeding process [4].

In this work, we show that cascaded four-wave mixing (FWM) [5] and Bragg scattering in an optical fiber [6] can be identified as the mechanism behind the dynamics of the resonant seeding of MI. Specifically, we approximate a picosecond pulse pump by an appropriate set of two distinct $\mathrm{CW}$ pumps and compare their corresponding MI spectra to understand the physical picture. A critical condition to excite the effect is finally discussed.

\section{Physical picture and experiment}

The use of fiber frequency comb brings great advantage to the description of some dynamics in conventional SC generation [5,6]. Using this approach to identify particular nonlinear dynamics in the seeded MI, we firstly consider the case of two CW pumps at frequencies $\omega_{\mathrm{p}}, \omega_{\text {-p }}$ seeded by a weak CW probe at frequency $\omega_{\mathrm{s}}$ whose schematic of the combination of the cascaded FWM and Bragg scattering is shown in Fig. 1(a). We analyze this nonlinear dynamic picture in three regions of the frequency domain. Region A is caused by the local cascaded FWM between two CW pumps, whereas region B is governed by the Bragg scattering between the seed and the pumps. Distinct sidebands separated by a pump frequency detuning $\Delta$ will be created localizing around the mutual center frequency $\omega_{\mathrm{c}}$ of the pumps in the region A and the seed frequency in the region B. FWM between the pumps and the seed leads

(a)

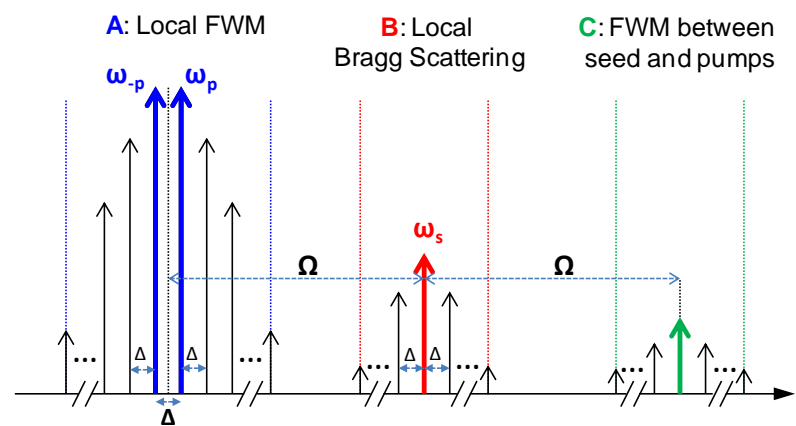

(b)

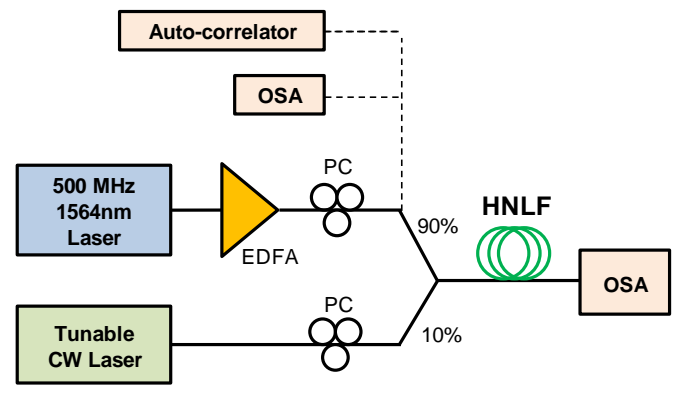

Fig. 1. (a) Frequency configuration of the seeded MI. (b) Experimental setup. $\Delta$ : pump frequency detuning. $\Omega$ : frequency distance between seed and center position of two pumps. PC: polarization controller. OSA: optical spectrum analyzer. 
to the growth of FWM signal at the center frequency of region C, and sequentially distinct sidebands will occur around this signal in region $\mathrm{C}$ as a result of the Bragg scattering between the new growing FWM signal and the pumps. The key nonlinear process of the resonant seeding effect in MI happens in region B and C. When the seed frequency coincides with the MI gain peak frequency, the seeding probe will get a largest gain and its power is amplified rapidly resulting in the power increase of Bragg scattering and FWM sidebands in the regions B and C.

To demonstrate the same dynamic picture appears in the case of quasi-continuous pulses with continuous spectral envelopes, we conduct a CW-seeded MI experiment on a picoseconds pulse as shown in Fig. 1(b). 4-ps input pulses at $1564 \mathrm{~nm}$ and repetition rate of 500MHz are amplified and combined with a CW seed probe at 1553 $\mathrm{nm}$ via a 90:10 coupler before injecting into a 60-m highly nonlinear fiber (HNLF). The HNLF has a nonlinear coefficient $\gamma$ of $10.5 \mathrm{~W}^{-1} \mathrm{~km}^{-1}$ and the second dispersion parameter $\beta_{2}$ of $-2 \mathrm{ps}^{2} \mathrm{~km}^{-1}$. The input peak powers of the pulsed pump and the $\mathrm{CW}$ probe are set to be $7 \mathrm{~W}$ and $0.5 \mathrm{~mW}$ respectively so that the output spectra is in the MI regime. Output seeded MI spectrum recorded by an optical spectrum analyzer (OSA) is shown in Fig. 2(a) (bold blackcontinuous line). We approximate the pulses by two $\mathrm{CW}$ waves separated by $\Delta=0.1 \mathrm{THz}$ and simulate numerically the MI spectrum using generalized stochastic nonlinear Schrödinger equation (GNLSE). An excellent agreement of the simulation MI spectrum for the two CW pump waves with the experimental MI spectrum for the picosecond pump in Fig. 2(a) and the observation of the seeded MI schematic explained associated with the dynamic regions A, B and C in Fig. 1(a) demonstrate that seeded MI of quasi-continuous pulses can be interpreted physically by fiber frequency combs and thus confirm our predicted physical picture (Fig. 1(a)). Particularly, local cascade FWM of two CW pumps in region A corresponds to the self-phase modulation of ps-pulses and the $n^{\text {th }}-\mathrm{FWM}$ between two CW pumps and the seed in region C corresponds to the $(n+1)^{\text {th }}$ side-lobes in the ps-pulses case. Naturally, to satisfy the phase matching condition of this FWM, the resonant seeding frequency detuning $\Omega_{0}$ must be outside the SPM region. This leads to a critical condition for the seeding effect: $\Omega_{0}=\left(2 \gamma P_{0}|| \beta_{2} \mid\right)^{1 / 2}>\mathrm{BW}_{\mathrm{SPM}}$, where $\mathrm{BW}_{\mathrm{SPM}}$ is the bandwidth of the SPM region. This condition is demonstrated in Fig. 2(b) that represents a false-color plot of the $-35 \mathrm{~dB}$ MI bandwidth ratio $r$ between the seeded and unseeded cases when the input peak power $P_{0}$ and the fiber length $L$ are varied. We can see that, for given $\beta$ and $\gamma$, there exists a critical power $P_{c r}$ in which no seeding effect can be observed regardless of the fiber length. This critical power $P_{c r}$ of $2.6 \mathrm{~W}$ shown in Fig. 2(b) is in good agreement with the one calculated using the above formula.

(a) Seeded MI spectrum

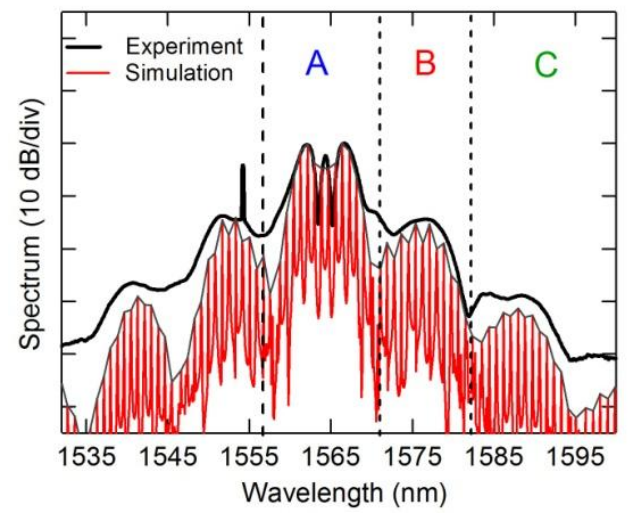

(b) -35 dB MI Bandwidth ratio between the seeded and unseeded case

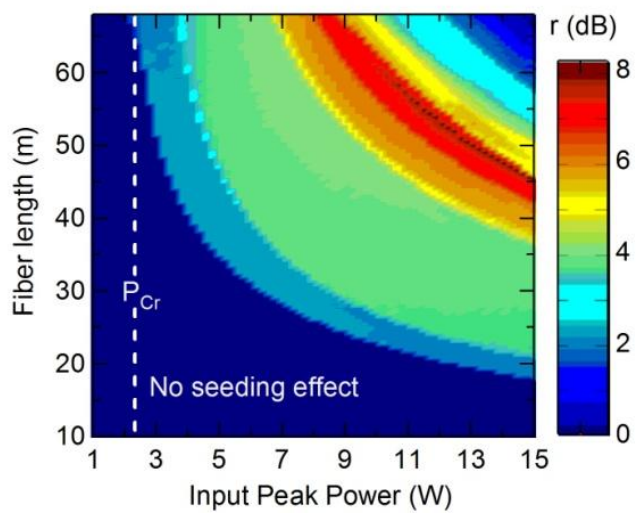

Fig. 2. (a) MI spectra seeded by CW probe at $1553 \mathrm{~nm}$. (b) $-35 \mathrm{~dB}$ MI bandwidth ratio between the seeded and unseeded case.

\section{Conclusions}

We demonstrate that the resonant seeding in MI on a quasi-continuous picoseconds pulse can be interpreted physically in terms of the cascaded FWM and Bragg scattering in fiber frequency combs. We explain why the MI spectrum bandwidth can be enhanced dramatically by seeding an extremely weak seed and finally report a critical condition to realize this effect.

\section{References}

[1] D. R. Solli et al., "Optical rogue waves," Nature 450, 1054 (2007).

[2] K. K. Y. Cheung et al., "Manipulating supercontinuum generation by minute continuous wave," Opt. Lett. 36, 160 (2011).

[3] D. M. Nguyen et al., "Incoherent resonant seeding of modulation instability in optical fiber," Opt. Lett. 38, 5338 (2013).

[4] J. M. Dudley et al., "Harnessing and control of optical rogue waves in supercontinuum generation," Opt. Express. 16, 3644 (2008).

[5] M. Erkintalo et al., "Cascaded Phase Matching and Nonlinear Symmetry Breaking in Fiber Frequency Combs," Phys. Rev. Lett. 109, 223904 (2012).

[6] Y. Q. Xu et al., "Cascaded Bragg scattering in fiber optics," Opt. Lett. 38, 142 (2013). 